\title{
eJRIEPS
}

Ejournal de la recherche sur l'intervention en éducation physique et sport

32 | 2014

Varia

\section{Le catch en EPS : une pratique physique originale adaptée aux élèves?}

Frédéric Loyer et Éric Dugas

\section{OpenEdition}

Journals

Édition électronique

URL : http://journals.openedition.org/ejrieps/2050

DOI : 10.4000/ejrieps.2050

ISSN : 2105-0821

Éditeur

ELLIADD

\section{Référence électronique}

Frédéric Loyer et Éric Dugas, «Le catch en EPS : une pratique physique originale adaptée aux

élèves ? », eJRIEPS [En ligne], 32 | 2014, mis en ligne le 01 avril 2014, consulté le 03 octobre 2019.

URL : http://journals.openedition.org/ejrieps/2050 ; DOI : 10.4000/ejrieps.2050

\section{(c) (1)}

La revue eJRIEPS est mise à disposition selon les termes de la Creative Commons Attribution 4.0

International License. 
Le catch en EPS : une pratique physique originale adaptée aux élèves ?

Frédéric Loyer* \& Éric Dugas**

* Laboratoire CesamS, EA 4260, Université de Caen, France

** Laboratoire LACES, EA 4140, Université de Bordeaux, France.

\section{Résumé}

En Education physique et sportive (EPS), les avis sont contrastés concernant l'effet cathartique des sports de combat comme un moyen, parmi d'autres, de substituer aux comportements violents et indésirables des "formes équivalentes d'agression " qui soient socialement acceptables (Elias \& Dunning, 1994). Pourtant, une pratique de combat singulière pourrait participer au "mieux-être " interactionnel des élèves ; il s'agit du catch. Effectivement, l'analyse des structures de cette activité physique révèle une liberté d'action laissée aux participants (transgression complice des règles, jeux de rôles préétablis).

Ainsi, un cycle original de "catch-scolaire " en EPS est proposé à une classe mixte de lycéens $(n=24)$. L'observation non participante des séances et les entretiens réalisés avec certains élèves (difficiles, introvertis, etc.) dévoilent que la pratique offre l'occasion de vivre des situations relationnelles insolites et fortement diversifiées. Si bien que le jeu de simulacre (mimicry) donne à l'adolescent la possibilité de définir sa propre structure du jeu et lui permet d'accéder à une régulation de ses conduites d'agression et de violence. Il participe ainsi à l'acquisition de compétences relationnelles et émotionnelles.

Mots-clés: sports de combat, catch, compétences relationnelles et émotionnelles, violence, agressivité.

\section{Introduction}

Les modifications, tant psychiques que physiologiques, liées à l'adolescence font naître chez le jeune des sentiments parfois douloureux. La violence, dont la forme la plus 


\section{eJRIEPS 32 avril 2014}

répandue est l'agression verbale, constitue, pour le plus grand nombre, le mode de communication principale (Dervaux \& Pain, 2006 ; Moatti, 2000). Au-delà du verbe, la violence physique est, elle aussi présente. Par exemple, les garçons sont davantage victimes de violences physiques alors que les filles sont plus confrontées aux violences sexuelles (enquête européenne ESPAD, 1994). Ces dernières années les médias se font aussi régulièrement l'écho d'actes de violences parfois graves se déroulant au sein même des établissements scolaires, ou dans leur périphérie immédiate. Les faits de violence renvoient communément à l'ensemble des actes caractérisés par des abus de la force physique, ou par des relations d'une extrême agressivité menaçant directement l'intégrité physique ou morale des personnes (Marzano, 2011).

Dans la sphère scolaire, ces actes se manifestent sous la forme de dégradations matérielles, d'incivilités, d'insultes, de menaces, de racket, de racisme... Malgré les campagnes de sensibilisation, les harcèlements peuvent aller jusqu'à des cas extrêmes (Debarbieux, 2012). Pour illustration, au cours de l'année scolaire 2011-2012, les établissements publics du second degré ont déclaré 13,6 incidents graves pour 1000 élèves, soit 1 point de plus qu'au cours de la période précédente, d'après le Système d'Information et de Vigilance sur la Sécurité scolaire (MEN-MESR-DEPP, enquête Sivis, 18 novembre 2012).

Face à cette inflation inquiétante de comportements déviants, la lutte contre la violence en milieu scolaire devient une priorité gouvernementale (circulaire $n^{\circ} 2006-125$ du 168-2006). Cependant, afin de lutter contre la violence au sein des établissements scolaires et de leurs abords, les pouvoirs politiques se tournent aussi vers le sport, et ce depuis quelques décennies déjà (Loi n̊4-610 du 16 juillet 1984 relative à l'organisation et à la promotion des activités physiques et sportives). Plus récemment, un des objectifs forts des derniers programmes d'éducation physique et sportive (EPS) pour les lycées d'enseignement général et technologique (Bulletin officiel spécial $n^{\circ} 4$ du 29 avril 2010) est celui d'amener l'élève à une gestion de ses conduites agressives et de son incivilité. Ainsi, le sport et l'éducation physique à l'école seraient sollicités, dans le cadre d'une action cathartique, d'une rééducation corporelle et morale et d'une éducation civique, pour contribuer à favoriser l'insertion et la réussite scolaires. 


\section{eJRIEPS 32 avril 2014}

Plus particulièrement, les sports de combat sont souvent privilégiés comme moyen permettant de canaliser son agressivité à travers la maîtrise des gestes, le respect des règles et de l'adversaire, ainsi que le contrôle des émotions (Carnel, Masschelein \& Boutoille, 2008). Plus largement, dans les sociétés civilisées où les états d'excitation et de tension sont constamment refrénés, le sport serait lié à la libération contrôlée des émotions (Elias \& Dunning, 1994). Les sports de combat apparaissent communément comme un moyen de substituer aux comportements violents et indésirables des «formes équivalentes d'agression » qui soient socialement acceptables. Dans cette perspective, l'éducation physique et sportive (EPS) construit des projets éducatifs en accordant aux pratiques de combat une fonction de décharge cathartique liée à l'expression possible de l'agressivité dans un cadre convenu et respectueux des autres. D'ailleurs, des recherches, notamment celles de Nosanchuk (1981) ; Lamarre et Nosanchuk (1999), concluent à des résultats allant dans le sens d'une catharsis de l'agressivité associée à la pratique d'arts martiaux (judo et karaté) privilégiant un idéal de vie et le respect de l'adversaire.

1.1. L'effet cathartique des sports de combat en questions Cependant, l'hypothèse de l'effet cathartique des sports de combat a donné lieu à des résultats contrastés et non généralisables. Sur le plan psychologique, les résultats obtenus par Pfister (1985), il y a déjà quelques décennies, vont dans le sens d'une augmentation de l'agressivité chez des élèves ayant suivi un cycle de lutte. Par ailleurs, selon Carrier (1992) mais aussi Santschi (1993), les sports de combat sont source de développement des comportements d'agression car ils autorisent le contact physique dans une relation d'affrontement. Quant à Bredemeier, Shields, Weiss et Cooper (1986 et 1987), ils mettent en évidence que les pratiquants de sports, à forts contacts physiques, présentent une plus grande tolérance à l'égard des comportements agressifs, et sont eux-mêmes plus agressifs dans la vie quotidienne. Dans cette perspective, Foster (1997) n'enregistre pas de variation de colère après dix semaines d'entraînement en aïkido et en karaté.

Plus récemment, Reynes et Lorant (2003) font ressortir que la pratique du judo s'accompagne d'un effet bénéfique sur la diminution d'agressivité et de l'amélioration 


\section{eJRIEPS 32 avril 2014}

de la maîtrise de la colère uniquement pour les filles. Après plusieurs recherches expérimentales, Collard (2004) suggère que le sport, et surtout les sports de combats, façonnent les comportements agressifs sans pour autant les réduire. Celui-ci note que, dans de nombreuses villes où l'utilisation du sport est venue s'insérer dans les projets d'enrayement de la violence, on a vu la délinquance au mieux stagner, au pire augmenter.

Par ailleurs, la recherche de Sébastien Guilbert (2009), révèle que les représentations de la violence dans le sport sont loin de traduire la réalité culturelle et technique des disciplines. Ainsi, peut-on entendre certains jeunes annoncer vouloir faire du judo ou du karaté pour apprendre à se battre ou être plus efficaces dans les bagarres ! Dans une autre étude, l'exemple fourni par un professeur de judo de l'Association Sportive d'un club de la région parisienne abonde dans ce sens: son club mène une action éducative en 2006, dans une cité réputée difficile, où la délinquance juvénile est réelle, il relate que lorsqu'il a « expliqué le principe du judo aux adolescents, au début ils n'y voyaient qu'une technique pour se battre.» «Ils pensaient également pouvoir me battre. Je leur ai prouvé l'inverse à leur grand étonnement. » (Rapport annuel du conseil national des activités physiques et sportives remis au ministère de la santé, de la jeunesse et des sports, décembre 2007, p. 45).

Enfin, plus proches de nos préoccupations scolaires, les résultats d'une expérience de type avant/après en EPS dévoilent que les groupes expérimentaux, dont les séances d'EPS sont accomplies sous la forme d'opposition simultanée, ont tendance à accentuer l'agressivité de l'individu agissant. A contrario, les groupes ayant réalisé des activités en solo et en coopération paraissent développer moins d'agressivité après coup (Dugas, 2011).

En synthèse, il apparait que si la pratique des sports de combat permet sans nul doute une catharsis des tensions physiologiques, un drainage énergétique (dépense énergétique élevée due à l'importance de la durée et de l'intensité des exercices de combat), elle ne permettrait pas pour autant une diminution des conduites agressives des pratiquants dans la vie quotidienne. En somme, comme le stipule très justement Debarbieux, le sport doit être perçu « comme l'élément d'un projet global d'éducation et 


\section{eJRIEPS 32 avril 2014}

non comme le remède préventif qui permettrait après son administration d'enseigner en étant débarrassé de la violence » (Debarbieux, 2011, p. 1).

Si les sports de combat situant l'opposition au centre des apprentissages font débat quant à leurs «potentialités » éducatives, certaines pratiques peuvent se dérouler sous la forme d'une coopération et amener des résultats intéressants sur le plan socioaffectif: contrôler ses émotions (peur, colère, agressivité), développer des attitudes de respect, améliorer ses relations de coopération. À cet effet, Michel Calmet (2006a) s'est intéressé au Kendo, le présentant dans le cadre scolaire comme un jeu de touches, de feintes et de relations : « il s'agit bien de toucher l'autre dans le cadre de confrontations adaptées mais en respectant des équilibres physiques (contrôler la touche : elle doit être "posée" à l'impact) et sociaux (respecter l'autre) » (Calmet, $2006 b$, p. 2). Dans la même veine, nous nous sommes intéressés à une pratique de combat originale, le catch, comme outil d'apprentissage des compétences relationnelles et émotionnelles permettant de pouvoir mieux comprendre et vivre l'autre, facteurs de diminution du comportement agressif. Les compétences émotionnelles passent par la régulation des émotions qui demande à séparer les réactions automatiques des émotions, comme la bagarre en cas de colère (Friedlmeier \& Holodynski, 1999). L'objectif devient ici de dépasser des émotions négatives et d'être capable de reconquérir une attitude positive.

Comme le révèle Terrisse (1996), dans les années quatre-vingt-dix, les didacticiens spécialistes des sports de combat de préhension s'accordaient sur l'utilisation de la variabilité du rapport d'opposition duo-duel ou avec-contre (Barbot, 1989 ; Brousse, 1993 ; Bui-Xuan, 1989). Une des modalités de variation reposait sur la fixation des rôles d'attaquant ou de défenseur et sur leur articulation (enchaînement de rôles). La structuration du couple à l'intérieur de la situation d'affrontement va se construire progressivement en jouant sur la difficulté du contenu présent à l'intérieur des tâches, à travers le degré d'opposition et la hiérarchisation des séquences technico-tactiques proposées. Même si la technique doit être appréhendée en fonction de l'adversité et non plus pour elle-même (Gresser, 1992), l'abord d'une étape technique paraît inévitable et reste souvent valorisée (Brousse, 1994). 


\section{eJRIEPS 32 avril 2014}

Si notre champ de recherche, réalisé en EPS, utilise certains éléments de la démarche clinique (entretiens), pour autant il ne recourt pas à l'interprétation profonde des intersubjectivités des sujets et de leurs discours, ni ne prend en compte l'implication du chercheur (Terrisse \& Carnus, 2009). Le cadre théorique du travail présenté est plutôt celui de « l'action située » (entre autres, Greeno, 1998 ; Kirshner \& Wisto, 1997 ; Lave, 1988) qui analyse l'activité humaine comme un accomplissement pratique, singulier, situé socialement et culturellement. L'expérience s'inscrit surtout dans le domaine de la recherche en « anthropologie cognitive située », à l'intérieur de laquelle il est accordé une priorité à l'expérience vécue, en décrivant les actions que les acteurs réalisent et les significations qu'ils leur donnent (Durand, 2001 ; Gal-Petitfaux \& Durand, 2001; Schubauer-Leoni, 2008 ; Sensevy, 2007 ; Cizeron, 2010). L'activité est utilisée comme moyen d'enseignement, permettant de transmettre et enseigner de nombreux savoirs aux élèves : technique, stratégique, sécuritaire, éthique, culturel, méthodologique (Loizon, 2008). Les objectifs de travail dépassent le « savoir combattre », le développement de compétences psychosociales. Savoir gérer le conflit, le stress, exprimer ses émotions et les gérer, travailler ensemble, se respecter sont ici essentiels (Simard et Jourdan, 2008).

Comme le note Bui-Xuan (1986), les sports de combats de préhension sont divers, ils ont des histoires, des significations sociales des modalités de pratiques qui ne peuvent qu'amener à les différencier. Les règles spécifiques de chaque activité définissent un ensemble de contraintes qui orientent des comportements propres, une motricité particulière. Ainsi, le catch prend appui sur des techniques de combat dans une relation d' « opposition complice » entre deux ou plusieurs acteurs, il peut se définir comme une « lutte-spectacle » où il s'agit de simuler le fait d'imposer physiquement un état corporel que l'autre refuse dans le but de susciter l'émotion auprès du public. La technique réinventée, voire inventée, appartient à l'élève (seul l'intégrité physique doit être respectée). L'histoire et la culture du catch dévoilent que les techniques correspondent à un savoir-faire individuel qui se développe depuis la fin du XIXème siècle. Certaines prises portent déjà le nom à cette époque du lutteur qui en est le meilleur spécialiste : le « tour de tête » d'Arpin, le « tour de hanche » à la Paul Pons, le 


\section{eJRIEPS 32 avril 2014}

contrôle au sol du « fond du sac » de Dumont, la « cravate » à la François Le Bordelais (Loyer, 2010).

Le travail envisagé autour du catch doit aider l'élève à se situer par rapport à ce qu'il fait ou dit à propos de la situation ou exercice proposé, afin de pouvoir mieux s'exprimer et maîtriser ses réactions émotionnelles et affectives. Cet enjeu fondamental ne peut être atteint à partir d'une conception traditionnelle, ni par une pédagogie classique des sports de combat (démonstration par l'intervenant d'une prise suivie de sa parade et de sa riposte) en utilisant une relation frontale de maître à élèves. Les modalités d'investissement et de construction de la pratique par le jeune lui-même sont essentielles. À travers le catch, l'élève-acteur détermine par son activité les éléments de l'environnement avec lesquels il interagit et construit à chaque instant sa propre situation.

Après avoir exposé l'intérêt pour un adolescent en construction d'une telle pratique en EPS, nous définirons la structure de l'activité autour de l'identification de ses caractéristiques. L'étude est conduite en collaboration avec un enseignant d'EPS volontaire pour recevoir le chercheur et proposer un cycle de catch à une classe de lycéens. Le catch est une activité physique sportive et artistique (APSA) spécifique à l'établissement. L'activité est validée (contenu, objectifs et organisation prévue) après présentation et acceptation du projet au chef d'établissement par le chercheur : période et durée, classe et enseignant concernés. Nous présenterons la méthodologie employée et les effets obtenus auprès de ce public afin de pouvoir répondre à cette question : le catch-scolaire contribue-t-il au développement de relations sociales et socioaffectives plus harmonieuses avec autrui ?

\section{Les élèves et la pratique du catch}

\subsection{Se poser en s'opposant}

Comme le note Yves Morhain (2001), le jeune qui sort des règles ou des interdits qu'imposent les conventions humaines, s'exile de la communauté, la défie du dehors, après avoir transgressé, outrepassé les frontières qui déterminent les normes qu'elle s'est donné. De la même façon, alors que l'adolescent se rebelle d'abord bien souvent 


\section{eJRIEPS 32 avril 2014}

contre la tentative d'imposition d'un ordre prédéfini, il vit comme une contrainte supplémentaire les règles d'une pratique sportive de combat qui lui sont soumises, accentuant parfois ces réactions de violence (Wargo, 2007). L'affirmation de soi nécessite un remaniement de l'agressivité qui ne soit pas destructeur et répond ainsi à un double besoin : celui d'actualiser son identité et celui de confirmer cette identité par l'autre (Meirieu, 2009). La diminution des comportements violents passe par le développement des comportements citoyens qui sont une conquête permanente, résultats d'expériences, de débats, de conflits (Meirieu, 2008). Ainsi, au-delà de la canalisation immédiate de l'agressivité dans des voies socialement acceptables, et en s'appuyant sur l'acquisition de compétences motrices, lutter contre la violence suppose aussi une éducation en actes, une sorte de « construction interactive par l'expérience » (Debarbieux, 2008, p.170). En somme, transformer les violences pour faciliter la bienveillance interactionnelle et l'empathie (Favre, 2007). Ceci, à condition d'innover pour créer la rencontre qui fait sens, la violence de l'adolescent n'est pas une fatalité. De fait, une identification des facteurs explicatifs des comportements violents ainsi qu'une compréhension du contexte dans lesquels ils se développent par l'enseignant et l'élève sont nécessaires. Car rappelons, entre autre, que les victimes de harcèlement et de brutalité entre les murs de l'école sont confrontées à de l'anxiété, de la dépression, à une baisse de l'estime de soi ou encore à des malaises physiologiques ou psychosomatiques (Smith, 2002).

La pratique du catch - où la règle existe pour être transgressée et pour permettre de structurer le jeu de rôle lui-même - peut, selon nous, répondre en partie à la problématique complexe de l'adolescence où le malaise corporel associe pulsions agressives et désir d'autonomie (Coslin, 2005). Derrière la maîtrise des techniques de combat, supports au catch ou à la lutte jouée, l'objectif lié à la construction des rôles sociaux et la connaissance d'autrui peuvent trouver ainsi un sens particulier. Du fait de l'intérêt porté au catch, analysons cette activité physique et sportive si singulière et les conséquences potentielles de sa pratique sur les conduites des élèves. 


\section{eJRIEPS 32 avril 2014}

\subsection{Le catch à la loupe}

L'originalité du catch réside dans le fait d'être une pratique de groupe (2 à 6 individus) qui, par l'affrontement aménagé, attache une importance particulière aux comportements sociaux et aux rapports directement liés à la notion de « contact », spécifique à la maîtrise d'une premier niveau de pratique en lutte. Le catch est une version adoucie des sports de combat, il peut permettre un meilleur engagement des filles au regard de ces sports trop marqués « masculin » (Louveau \& Davisse, 1998). Or pour Butler, «le genre est toujours un faire, mais non le fait d'un sujet qui précéderait ce faire. »(Butler, 2006, p. 96). Ce faire pourrait se construire de façon dynamique dans l'apprentissage du catch scolaire.

La structure de l'activité englobe les possibilités de gagner, les déplacements dans l'espace, les configurations spatiales et temporelles, les relations avec le(s) autre(s) ... L'analyse du catch rendue ici a été réalisée grâce à l'observation de dix matchs de catch obtenue par la diffusion régulière des spectacles américains sur les chaines de la TNT : la victoire en catch s'obtient par «tombé » en maintenant les épaules de « l'adversaire » trois secondes en contact avec le tapis, en le forçant à abandonner sur une prise de soumission (étranglements, clefs), en le mettant knock out (perte de connaissance) ou en le projetant hors du ring plus de dix secondes. La transgression permanente des règles semble faire partie des rituels de l'activité et permet de construire un combat au scénario à rebondissements dont l'objectif est de déclencher l'enthousiasme du public. Effectivement, les règles d'un match sont utilisées pour simuler la compétition en désignant un vainqueur, mais surtout pour enrichir l'histoire d'un match. Les affrontements truqués, l'absence de catégories de poids, les règles continuellement transgressées, les techniques à l'apparence violente, constituent les traits caractéristiques du catch qui ne peut se ranger totalement dans la famille des sports de combat au sein desquelles les notions d'opposition et de duel strictement compétitif sont centrales (cf. tableau 1 ci-après). 
eJRIEPS 32 avril 2014

Tableau I. Traits caractéristiques du catch observés lors de dix rencontres télévisées.

\begin{tabular}{|c|c|}
\hline & Catch \\
\hline Structure & $\begin{array}{l}\text { Jeu à information « privée » } \\
\text { Jeu à } n \text { joueur : } 2 \text { à } 6 \\
\text { Jeu strictement coopératif } \\
\text { Jeu strictement déterminé }\end{array}$ \\
\hline $\begin{array}{c}\text { Temps } \\
\text { Comptabilité }\end{array}$ & $\begin{array}{l}\text { Temps variable } \\
\text { Codification peu claire } \\
\text { Un match peut se gagner par : tombé (décompte de trois); } \\
\text { soumission ; décompte à l'extérieur du ring (dix secondes); } \\
\text { disqualification }\end{array}$ \\
\hline $\begin{array}{l}\text { Espace } \\
\text { Terrain }\end{array}$ & $\begin{array}{l}\text { Plat et régulier - spécifique } \\
\text { Tracé et préparé (ring) mais délibérément non respect }\end{array}$ \\
\hline $\begin{array}{c}\text { Interaction } \\
\text { d'oppositions }\end{array}$ & $\begin{array}{l}\text { Au contact au corps à corps } \\
\text { Préhension } \\
\text { Percussion } \\
\text { Frappes par l'intermédiaire d'un instrument } \\
\text { Partenariat }\end{array}$ \\
\hline Violence corporelle & Simulée \\
\hline
\end{tabular}

Le catch apparait en tant que jeu présentant un réseau de communication et de rôles différents du duel symétrique qui caractérise les sports de combat. Dans une partie de catch les relations sont ambivalentes, chacun est en même temps partenaire et adversaire de certains autres, au gré des fantaisies des joueurs, des circonstances du jeu, des réactions du public (Loyer, 2013). Les élèves-catcheurs sont soumis à une règle de permutation systématique de rôle à rôle, chacun pouvant successivement endosser tous les rôles disponibles. Les règles et scénarii se construisent et se déconstruisent en permettant de vivre des situations relationnelles insolites et fortement diversifiées. Ainsi les situations offrent-elles la possibilité à l'élève de s'immerger dans un univers social éloigné du schéma dichotomique et stéréotypé des sports d'oppositions classiques. La construction, l'exécution et l'évolution des scènes de catch doivent révéler un élève qui accepte, partage, négocie, discute, argumente, 


\section{eJRIEPS 32 avril 2014}

critique, coopère, s'investit, collabore, soutient ou assiste ; en somme, elles peuvent révéler un élève-citoyen.

Ce que le catch est surtout chargé de « mimer», c'est un concept purement moral, celui de justice, car le combat oppose toujours un «salaud parfait » à un héros (Barthes, 1957). Le salaud est essentiellement un instable qui se réfugie derrière la loi quand il juge qu'elle lui est propice et la trahit quand cela lui est utile : « tantôt il nie la limite formelle du ring et continue de frapper un adversaire protégé légalement par les cordes, tantôt il rétablit cette limite et réclame la protection de ce qu'un instant avant il ne respectait pas »(Barthes, 1957, p. 22). Les catcheurs sont soumis à une règle de permutation systématique de rôle à rôle, chacun pouvant successivement endosser tous les rôles disponibles: le bon, le mauvais, le gentil, le méchant, le « salaud », le « souffre-douleur »... Les relations entre catcheurs apparaissent alors ambivalentes et de nature paradoxale, les «adversaires » peuvent se retrouver à un moment donné de la partie complice face à l'arbitre, partenaire et adversaire des uns ou des autres, au gré des fantaisies des joueurs, des circonstances du jeu, des réactions du public. Le catch repose sur la simulation d'un rôle, la création d'une illusion dramatique, la représentation d'un personnage. Seule l'apparence de la lutte sportive demeure; l'activité s'inscrit dans la définition donnée par Caillois (1958) des jeux de type « mimicry»: « devenir soi-même un personnage illusoire et se conduire en conséquence ». Le jeu de simulacre (mimicry), permis par la pratique du catch, serait ainsi susceptible de donner à l'élève la possibilité de pouvoir définir, tout en jouant, sa propre structure du jeu.

À partir de ce contexte d'action original, il apparaît que le catch fonctionne selon un modèle de partenariat où l'alternance permanente des statuts de « dominant/dominé » peut donner l'occasion à l'élève de s'immerger dans un univers social éloigné du schéma classique des sports strictement compétitifs, au sens de la « théorie des jeux » (Guerrien, 2002).

Comme déjà exprimé plus haut, le catch est spectacle, il repose sur la simulation d'un rôle, la création d'une illusion dramatique, la représentation d'un personnage et pourrait faciliter, in fine, le bien-être relationnel entre les élèves. 


\section{eJRIEPS 32 avril 2014}

\subsection{Les enjeux éducatifs du cycle catch}

Le catch-scolaire se situe à l'interface des champs de compétence propre à l'EPS visant à réaliser une prestation corporelle à visée artistique ou acrobatique (CP3), et cherchant à conduire et maîtriser un affrontement individuel ou collectif (CP4). Les objectifs éducatifs du cycle sont construits en concertation avec l'enseignant d'EPS, ils visent le développement des compétences propres à l'EPS, spécifiques aux sports de combat de préhension et d'ordre social et civique liées à la vie citoyenne. Contrairement au spectacle sportif, la vertu du catch est d'être un spectacle délibérément excessif. Mieux qu'en lutte sportive, les catcheurs passent tour à tour du statut de subordonnant à celui de subordonné, accentuant la révélation du pouvoir d'oppression que chacun peut exercer sur les autres.

L'élève doit apprendre à distinguer les situations d'opposition et de coopération afin de pouvoir utiliser la relation de partenariat, le duo et non le duel dans la construction d'un spectacle de catch demandant la maîtrise de connaissances, capacités et attitudes spécifique à la lutte. Plusieurs principes sont en effet à comprendre et à maîtriser en action : la notion d'utilisation de la force de l'adversaire, les principes d'immobilisation, les principes de retournements, les principes des techniques de projection vers l'avant sur deux appuis, vers l'arrière sur un appui. Savoir chuter dans les quatre directions (avant, arrière latérale droit et latéral gauche) est nécessaire avant la mise en situation des « jeux de rôle » luttés.

À travers le catch-scolaire, activité support de l'EPS, il est recherché prioritairement le développement des compétences relationnelles et émotionnelles permettant de mieux comprendre et vivre l'autre. II s'agit de maîtriser, comme individu et comme citoyen, les règles élémentaires de la vie en société et de les mettre en œuvre. Les liens recherchés avec le socle commun visent la compétence 6, «comprendre l'importance du respect mutuel, accepter les différences, participer à la réalisation d'une production collective », et la compétence 7 : « concevoir et mener un projet à son terme » (Bulletin officiel spécial $n^{\circ} 4$ du 29 avril 2010). Les compétences méthodologiques et sociales révèlent l'acquisition de méthodes, d'attitudes, de démarches réflexives. Elles se situent dans le respect des règles de vie collective, la maîtrise des émotions et 


\section{eJRIEPS 32 avril 2014}

l'acceptation du regard des autres. L'élève acquiert essentiellement des repères dans plusieurs domaines : les droits et les devoirs du citoyen, les notions de responsabilité et de liberté et le lien qui existe entre elles, les principes d'un État de droit.

Les professeurs d'EPS font souvent référence à la dimension physique qu'ils associent à la « gestion de la vie physique d'adulte » des programmes. Pour eux, c'est surtout le bienêtre physique qui compte avant tout. Par contre, ils ne font aucun lien avec l'éducation au choix, telle qu'elle est proposée dans la définition de la circulaire de 1998 : « L'éducation à la santé vise à aider chaque jeune à s'approprier les moyens d'opérer des choix, d'adopter des comportements responsables, pour lui-même comme vis-à-vis d'autrui et de l'environnement ».

Construire un projet d'action pour composer une scène luttée en tenant compte du thème proposé, ici «l'agression entre pairs », demande aux élèves de se conduire dans le groupe en fonction de règles, de codes, que l'on comprend et que l'on respecte. Coopérer, adopter des attitudes d'écoute, d'aide, de tolérance et respecter les autres pour agir ensemble et chercher de nouvelles idées afin de construire et faire évoluer un scénario est indispensable. Les élèves doivent être capable de montrer aux autres ce qu'ils proposent de faire, d'observer ce que proposent les autres, afin d'adopter à plusieurs un projet. En somme, une réflexion à travers une mise en œuvre des contraintes de la vie collective (liens entre liberté personnelle et contraintes de la vie sociale) est principalement poursuivie au cours du cycle. L'activité doit effectivement s'intégrer dans une activité sociale qui lui donnera une signification supérieure. Ainsi, la maîtrise de soi et de l'autre se fera toujours par rapport à un modèle de référence que le sujet construira progressivement avec le groupe. Cependant, si entrer dans l'APS catch permet d'accroître ses possibilités d'actions sur l'autre, les sujets doivent construire un espace commun d'affrontement, connaître leur corps et leurs limites comme ceux de leur adversaire...Autant de transformations difficiles à mettre en place chez l'adolescent en pleine transformation et à plus forte raison chez l'adolescent 
eJRIEPS 32 avril 2014

\section{Méthodologie}

3.1. Sujets et tâches en EPS

Le cycle d'apprentissage se déroule dans un lycée de province avec une classe terminale mixte de 24 élèves (10 filles et 14 garçons), dont l'âge moyen est de 17,5 ans. Les élèves appartiennent au même environnement socioculturel, de type populaire. L'enseignant d'EPS volontaire est un spécialiste de la lutte, titulaire d'un brevet d'état, il organise régulièrement les championnats académiques UNSS de cette discipline, il n'a jamais proposé le catch comme activité support à son enseignement ; âgé de 45 ans, il a 20 années d'ancienneté à l'Éducation Nationale. La plupart des élèves ont des difficultés scolaires. Aux dires de l'enseignant, l'EPS reste un moyen pour eux de se détendre et se défouler. Sur l'ensemble du groupe, il existe un certain nombre d'éléments perturbateurs dont certains ne semblent pas motivés par cette discipline et d'autres se caractérisent par un manque de confiance en eux.

Le cycle se déroule durant sept semaines et représente une durée totale de quatorze heures de pratique. II se situe entre la période des vacances de Noël et de février, après un cycle de handball et avant un cycle de course d'orientation.

L'objectif attendu en fin de cycle vise à composer et présenter un numéro collectif organisé autour d'un thème incorporant, à un jeu d'acteurs, des techniques de lutte simples. Autrement dit, à côté des objectifs de développement physique et moteur tels que l'amélioration de l'exécution motrice générale, la maîtrise de techniques issues de la lutte, ceux liés à la construction des rôles sociaux et le mieux-être social constituent l' axe éducatif central du cycle. La conduite d'un débat (inséré dans un combat simulé), l'engagement, l'entraide et la solidarité, l'empathie, sont autant d'attitudes nécessaires et citoyennes attendues des élèves dans la construction d'une meilleure "santé sociale » (Delignières, 2001).

Après concertation avec l'enseignant, quatre élèves aux profils et comportements caractéristiques du groupe-classe ont été retenus pour former un groupe de travail qui sera suivi pour la recherche menée. Les prénoms des élèves sont modifiés dans un souci d'anonymisation ; ils seront nommés: Laura, Mathieu, Victor et Théo. 


\section{eJRIEPS 32 avril 2014}

La démarche méthodologique utilisée relève d'une observation directe non participante et d'entretiens entre le chercheur et les élèves. À cet effet, trois types de données ont été recueillis pour cette étude: (a) des données d'enregistrement audio des quatre élèves et de l'enseignant autour de l'activité en continu et in situ, et ce à l'aide d'un dictaphone numérique USB ; (b) la description des comportements moteurs à l'intérieur d'un journal de terrain ; (c) des données d'enregistrement audio lors d'entretiens postséances réalisés par le chercheur avec les quatre élèves au cas par cas, afin de rendre compte de leur expérience individuelle (sociale et émotionnelle).

L'étude de cas est au cœur du cadre méthodologique employé et vise, d'une part, à décliner le plus précisément possible la personne, sa situation et ses difficultés personnelles, d'autre part à éclairer l'origine et la genèse de ses problèmes (Albarello, 2009). La nature des entretiens mis en place durant et après le cycle de travail, est de type « semi directif » par rapport aux thèmes de la socialisation et des identités sociales (exemples de thèmes /questions: la violence, le respect des autres, l'égalité filles/garçons, la coopération, l'injustice) ; Les stratégies d'intervention reposent essentiellement sur les relances, mais pas sur de nouvelles orientations. Elles permettent de prendre pour objet le « dire antérieur » des élèves (Blanchet et Gotman, 1992). Les buts et objectifs du cycle d'apprentissage sont expliqués aux élèves, mais pas le but/hypothèse de la recherche, de même pour l'enseignant. Enfin, tout n'est pas dans le discours ou les paroles, à travers l'analyse clinique, c'est aussi une attention au non-verbal, au non-visible, à l'écoute des silences et aux effets émotionnels. Ainsi, l'attitude méthodologique utilisée, propre à une approche de la psychologie clinique, consiste à relever aussi fidèlement que possible les manières d'être et de réagir des élèves aux prises avec une situation, à chercher à établir le sens, la structure et la genèse de la conduite, à déceler les conflits qui la motivent et les démarches personnelles qui tendent à résoudre ces conflits.

Les quatre élèves susnommés acceptent le protocole expérimental passant par l'enregistrement audio des situations et la participation à des entretiens post-séances et post-cycle. Des échanges entre l'enseignant et le chercheur ont lieu systématiquement après chaque leçon afin que la leçon suivante puisse se construire 


\section{eJRIEPS 32 avril 2014}

en concertation. Le dictaphone numérique USB (avec micro intégré) est posé sur une table à proximité du groupe d'élèves. L'outil présente une grande qualité sonore et possède la capacité de démarrer automatiquement en présence de sons ou de voix à proximité. Le port USB permet le transfert les données vers un ordinateur permettant un travail fiable. Les entretiens se sont déroulés après les leçons d'EPS entre 13h et 13h30. Ils ont consisté à demander aux élèves du groupe expérimental de décrire a posteriori ce qu'ils avaient fait, ce qu'ils pensaient, ce qu'ils avaient pris en compte pour agir, ce qu'ils avaient perçu ou ressenti.

Le recueil des données audio croisé avec les entretiens post-cycle d'apprentissage permettra de s'assurer de la réalité de la pratique évoquée (Arborio \& Fournier, 2010). Les résultats qui vont suivre permettent principalement de dégager les similitudes et distinctions enregistrées entre les quatre élèves et ce, tant sur le plan moteur que sur le plan du discours. Autrement dit, les conduites motrices et verbales étudiées permettront de révéler l'éventuel impact d'une telle séquence d'apprentissage fondée sur l'acquisition de compétences de type social et relationnel.

\section{Résultats de la recherche}

4.1. Analyse des comportements moteurs et des échanges verbaux L'étude effectuée autour du catch-scolaire s'inscrit tout au long du cycle ; nous rendons compte et analysons une séance située en début de cycle, milieu de cycle et fin de cycle.

Les situations et les thèmes de travail proposés aux élèves sont décrits afin de situer le contexte à l'intérieur duquel les données sont recueillies ( $a, b$ et $c$ ). L'analyse des comportements moteurs et des échanges verbaux ont pour but d'évaluer le développement des compétences sociales et émotionnelles à travers les capacités à : prendre des décisions, créer, critiquer, communiquer efficacement et habilement, montrer de l'empathie pour ses camarades, gérer son stress et ses émotions. Précisons ici qu'il existe plusieurs degrés d'empathie qui concernent principalement la sphère de l'affectivité (exprimée par la joie, colère..) et celle de l'intentionnalité (compréhension de la situation). Pour ce deuxième cas de figure, comprendre 


\section{eJRIEPS 32 avril 2014}

l'intentionnalité d'une personne demande d'explorer le contexte de l'action (Rimé, 2005). Car l'empathie est au cœur du jeu social (Tisseron, 2010). Et pour un observateur, il ne faut pas confondre l'empathie de la confusion empathique. Par exemple, Daniel Favre (2007) décline trois types d'empathie pour un test auprès d'élèves : l'empathie proprement dite (la représentation de ce que pense ou ressent l'autre), la contagion empathique (se laisser envahir par les émotions d'autrui), et la coupure émotionnelle (mise à distance d'émotions, incompréhension d'autrui).

La sécurité étant essentielle, l'enseignant débute le cycle à travers un échauffement des articulations et une progression d'exercices proposée à l'occasion de chaque séance afin d'habituer les élèves à baisser leur centre de gravité, à s'enrouler, à chercher le tapis avec le bas du dos sur une chute arrière, à rentrer et protéger sa tête sur une chute avant. Ce travail est réalisé sous différentes formes, en coopération, en opposition modérée. Rappelons que le catch-scolaire sous-tend un projet de communication qui prend appui sur des techniques de combat utilisées selon une opposition aménagée qui deviendra une opposition complice.

Les situations de déséquilibre et de projection demandent la construction de contrôles qui conduisent à des « techniques » en décalage (fixation poussée, fixation rotation) et sous forme de hanché. Une première situation s'inscrit dans ce sens afin d'aborder les contrôles bras intérieur, bras tête, bras dessus/dessous, pour passer dans le dos du partenaire-adversaire et l'entourer avec les deux bras à la taille, sans perdre le contact avec celui-ci. La réussite demande, entre autres, le développement des capacités de coopération : aide, solidarité et collaboration. La maîtrise des réactions affectives et émotionnelles est nécessaire et passe par la gestion des comportements agressifs, violents ou au contraire trop timorés.

Le comportement général observé va vers l'individualisme, le non respect des autres et des différences, se manifestant par des moqueries importantes, témoignant de microviolences réitérées. Les élèves, constituent ainsi un groupe de travail difficile, surréactifs en regard des stimuli de l'environnement humain, ils montrent des difficultés d'écoute et de concentration, le groupe est alors bruyant et le retour au calme difficile. 


\section{eJRIEPS 32 avril 2014}

L'enregistrement audio des propos informels des élèves lors de la situation montrent que derrière ces similitudes plusieurs profils se dégagent. Certains sont « perturbateurs », ils présentent des difficultés à respecter le « savoir vivre ensemble » et témoignent d'un manque de respect envers autrui. Les agressions physiques et l'utilisation de certains mots vulgaires et argotiques sont fréquents: «bouffons, guignol... » (Victor) ; «viens là que je te défonce... » (Victor), « je peux te donner des coups de pieds si tu veux...» (Théo). D'autres sont repliés sur eux-mêmes et manifestent un manque d'enthousiasme et d'énergie. Laura ne s'implique pas, elle refuse la situation : «je laisse tomber...Je vais m'entraîner à faire des roues... ». Mathieu paraît plus volontaire, il stimule ses camarades et montre de l'impatience «alors on commence quand? », il va ensuite s'assoir le long du mur et adopte un comportement «provocateur», «vous êtes nuls les gars...Laura tu es une vraie danseuse! ».

L'entretien post-séance réalisé par le chercheur a pour but de faire verbaliser les élèves autour de leurs comportements déviants.

«La situation proposée n'a pas bien fonctionné et provoqué beaucoup de désordre comment l'expliques-tu ?». À travers les réponses des élèves des points de convergences importants apparaissent. Les actions menées ne semblent avoir de valeur pour ces élèves que dans le cadre du groupe qui lui donne sa signification sociale, c'est-à-dire que l'adolescent ne réagit à ses actes que par rapport aux valorisations que le milieu humain, dans lequel il se trouve, élabore à travers les modèles implicites qu'il véhicule : «j'ai toujours la meilleure note quand on fait un cycle de rugby ou de hand...Je vais pas me laisser battre !» (Théo) ; «il me donne des coups d'épaule...Je fais pareils ...» (Victor). Cependant des différences existent, si certains élèves usent de la violence pour «protéger » leur statut de dominant ou de leader dans la classe d'autres se font remarquer quand ils ne le peuvent pas par leurs résultats : «je perds tout le temps...Je préfère m'entrainer en gym... J'assure... » (Laura).

De fait, l'objectif est de resserrer les liens affectifs entre les jeunes, permettant de passer d'un rassemblement d'adolescents difficiles à un groupe organisé autour de 


\section{eJRIEPS 32 avril 2014}

référents communs. L'enjeu vise à participer un tant soit peu à la transformation interne de l'adolescent en essayant de contribuer à la mise en place de nouveaux équilibres émotionnels. C'est à travers un investissement relationnel et affectif prenant source dans l'engagement actif des élèves à l'intérieur du groupe que la pratique peut contribuer à un bien-être ou «mieux-être » social. La réussite passe, entre autres, par le développement des capacités de coopération : aide, solidarité et collaboration. La maîtrise des réactions affectives et émotionnelles est nécessaire à travers la gestion des comportements agressifs et violents.

Au cours de la troisième leçon, l'enseignant regroupe les élèves et annonce qu'ils doivent élaborer par groupes de quatre un scénario à partir d'un événement de la vie quotidienne mettant en action un harceleur, une victime et deux témoins de la scène. Plusieurs issues doivent être imaginées, jouées puis présentées à l'ensemble de la classe.

Par le biais d'un travail collectif, il s'agit de construire un groupe solidaire, de faciliter l'expression de chacun, le questionnement, la volonté de réussir, et de faire émerger des idées dans un but de création. Le groupe observé montre une certaine excitation et semble enthousiasmé par le travail proposé. Une scène reproduisant une sortie en discothèque est très vite imaginée. Théo arrive avec Laura, sa copine, en la tenant par le bras, ils montent sur le ring et dansent. Victor dans la discothèque courtise Laura, il se moque de Théo et le bouscule. Une bagarre éclate, Laura essaie de défendre son camarade, Mathieu, le responsable du service d'ordre, arrive et ne sait pas comment réagir, les deux garçons sont évacués du lieu. Ainsi, la communication avec les autres, la recherche de nouvelles idées, la prise d'initiatives caractérisent l'évolution des conduites de ces élèves.

En parallèle, sans être dirigés exclusivement sur la maîtrise technique, les élèves acceptent plus facilement le travail des contrôles et développent la capacité à varier les formes et opportunités d'attaques directes en utilisant les tirades et les poussées de l'adversaire. Conseiller un camarade sur la meilleure façon de s'y prendre pour réussir caractérise les échanges du groupe « quand je te pousse tu dois m'attraper et me tenir le bras pour me faire tomber...» (Victor à Théo) ; «tu me tires et j'accélère 


\section{eJRIEPS 32 avril 2014}

pour te barrer la jambe sur l'arrière... » (Mathieu à Laura). L'opposition mieux maitrisée devient alors complice, les élèves ajoutent spontanément des mimiques et jeux de scènes, qui remplacent les rires jusqu'alors fréquents dans le déroulement du scénario.

Comme envisagé avec l'enseignant, des débats sont aussitôt mis en place autour du jeu de catch et l'éducation à la citoyenneté, tels que le respect des autres, l'égalité filles/garçons, la coopération, l'injustice, la camaraderie. II s'agit dès lors d'aider les élèves à réfléchir sur certains de leurs comportements déviants, afin de leur apprendre à mieux se connaître, et donc, à terme, de mieux se contrôler. Les

échanges verbaux type dilemmes moraux (expliqués un peu plus loin) sont essentiels, il s'agit d'aider l'élève à se situer par rapport à ce qu'il fait ou dit à propos de la situation ou exercice proposé, afin de pouvoir mieux s' exprimer et maîtriser ses réactions émotionnelles et affectives.

«Quelle est la relation garçons - fille dans le scenario proposé ? (Enseignant) : «je suis la fille...je suis venue avec mon copain ...j'ai rien à dire... » (Laura) ; « encore heureux!» (Mathieu), des rires suivent dans le groupe classe..., « de toute façon en boite c'est toujours autour des filles qu'il y a des problèmes... » (Théo).

L'enseignant suggère alors : « est-ce qu'il est possible d'imaginer une autre évolution au scénario ? ». Une élève prend la parole : «les filles aussi peuvent se défendre! »; «Laura peut choisir le copain avec qui elle veut passer la soirée... » (Victor) ; « on peut essayer » (Mathieu).

Les élèves témoignent d'un fonctionnement collectif plus important, ils coopèrent et adoptent des attitudes d'écoute pour agir ensemble et élaborer un nouveau projet. Le groupe envisage, propose et met en scène une seconde issue, le statut de Laura évolue : lorsque Victor, le harceleur, et Théo, le harcelé se retrouvent dehors, Laura sort de la discothèque, elle met à terre Victor pour retourner danser avec Théo.

L'entretien post-séance, réalisé par le chercheur, révèle une évolution émotionnelle des élèves qui communiquent plus aisément, montrent de l'empathie et s'interrogent sur la montée de la violence «gratuite»: «comment avez-vous ressenti l'évolution du scénario et de sa mise en scène ? (Chercheur) : «ça a changé ma façon de voir les 


\section{eJRIEPS 32 avril 2014}

choses, moi aussi je peux décider... » (Laura) ; «Laura avait l'air vraiment contente, j'ai bien aimé lorsqu'elle a levé les bras au ciel après avoir balancé Victor... » (Mathieu).

Comme évoqué dans la présentation de la méthodologie, la relance de l'entretien s'effectue autour d'un thème en relation avec les réponses des élèves, ici l'égalité filles/garçons : à la question « la camaraderie est-elle possible entre fille et garçon ? Le respect de l'individu n'est-il pas essentiel ?» (Chercheur), les réponses sont bigarrées : « souvent on cherche la bagarre, mais c'est pour frimer devant les potes...et les filles » (Victor), «...mais la loi du plus fort n'est pas toujours la meilleure... » (Laura), « c'est bien que Laura ait pu prendre les choses en main...» (Mathieu). Si les élèves soulignent l'importance du respect mutuel et du dialogue, des divergences liées au genre demeurent. Par exemple, Théo imagine encore difficilement une fille capable de se défendre et se situant sur un pied d'égalité avec un garçon : «c'est sympa qu'on puisse raconter des histoires, mais moi je ne me laisserai pas battre par une fille... ». Au cours de la sixième séance, l'objectif est de poser en acte la notion de dilemme offrant aux élèves plusieurs alternatives menant à des résultats différents, ceci afin de permettre l'installation de discussions et de débats. Ce type de démarches se retrouve fréquemment dans les enseignements distillés au Canada, sous forme de dilemmes moraux (Dionne \& Saint-Martin, 2005). Le but est de faire évoluer les premiers scénarii en incluant un « spectateur » qui assiste à l'agression. Celui-ci doit proposer et jouer avec ses camarades une scène parmi plusieurs possibles : aide à l'agresseur, aide à l'agressé, indifférence...Le groupe observé fait évoluer la scène, Théo arrive avec Laura, ils dansent mais très vite il se comporte mal, fait n'importe quoi et embête Laura. Mathieu un client s'interpose et une bagarre éclate. Victor, un ami de Théo déjà présent dans la discothèque, observe les comportements, mais il ne bouge pas.

La leçon se termine sur ce scénario imaginé et présenté à la classe. Lors de l'entretien post-séance, nous confrontons les élèves aux notions de dilemme social et individuel. Un dilemme social existe lorsque l'intérêt de la collectivité est en conflit avec l'intérêt individuel (Eber, 2006). En recherchant leur intérêt personnel, les individus convergent 


\section{eJRIEPS 32 avril 2014}

la plupart du temps vers la solution la moins bénéfique pour la collectivité. Sur le plan expérimental, des comportements coopératifs peuvent cependant émerger.

«Qu'est-ce que Victor aurait dû faire ? », «Est-il facile de se mettre d'accord sur le «meilleur choix» à faire? Pourquoi ?» (Chercheur). Les élèves arrivent progressivement à distinguer «pouvoir » ou «être capable de faire » et « devoir faire ». «C'est pas évident, je suis le copain de Théo, même si c'est lui qui commence l'embrouille, j'ai pas envie de balancer un pote... », « j'aurais pu m'interposer entre les deux...C'est ça l'amitié !» (Victor) ; « Mathieu se comporte bien ...Victor pourrait aller discuter avec son copain pour le raisonner...» (Théo) ; « de toute façon on est libre de juger et de faire des choix ...» (Laura).

Le chercheur relance l'entretien autour de la notion des valeurs sociales et demande alors «Quelle(s) valeur(s) peut (vent) guider le choix de celui qui assiste à l'agression $? »$ :

Le cheminement réflexif des élèves évolue dans le même sens : Laura discerne ce qu'il convient de faire, dans telle ou telle situation, quand on est un être humain conscient de ses droits et des valeurs à préserver: "dans cette situation c'est Théo qui ne respecte pas les autres et doit être mis dehors... »; « il faut mesurer la part de responsabilité de chacun... » (Mathieu) ; «le plus intelligent ça serait que mon copain sorte dehors avec moi...C'est moi qui ai cherché l'embrouille... » (Théo).

Ainsi, les adolescents sont confrontés à la responsabilité qui incombe à chacun de départager un tant soit peu le bien et le mal, en vue de contribuer au mieux-être et au mieux-vivre de l'individu et de la collectivité. Les élèves envisagent leurs rôle et place dans le groupe de façon différente; leurs représentations des relations sociales quittent progressivement le pôle uniquement conflictuel et individuel pour envisager la possibilité de rapport de coopération et d'entraide.

En résumé, la pratique du catch a permis de donner du sens in vivo à la recherche d'une éducation à la citoyenneté, seule condition pour obtenir un résultat à long terme concernant les comportements et les attitudes des adolescents. Le principe de faire l'expérience de la démocratie à l'école, c'est-à-dire de vivre en actes l'exercice de la citoyenneté en faisant de la classe une sorte de microsociété, est essentiel. C'est 


\section{eJRIEPS 32 avril 2014}

d'ailleurs ce que nous allons chercher à vérifier maintenant à travers la réalisation d'entretiens post-cycle, avant de discuter les résultats enregistrés au sein de cette recherche.

\section{Entretiens post-cycle avec les élèves}

Les entretiens menés après le cycle « catch » vont nous permettre de confirmer ce que l'observation et l'analyse des conduites motrices et verbales ont laissé entrevoir : outre le plaisir de pratiquer des techniques et tactiques sportives, dont l'issue est jouée d'avance, ils ont "osé » le discours, le dialogue avec l'autre. L'accès aux nuances profondes entre opposition et coopération permet de faire évoluer progressivement des situations dans lesquelles le dialogue est possible.

«Que pensez-vous du cycle catch sur le plan relationnel vis-à-vis de vos camarades ? »(Chercheur).

À cette question liminaire, se succèdent des avis globalement positifs envers le vécu de ce cycle original en EPS, mais ils sont toutefois emprunts de fines distinctions « c'est intéressant qu'on puisse discuter pendant et à la fin des séances pour faire avancer les scénarios » (Laura). Pour Théo, «à travers la construction et l'évolution des scènes la discussion est obligatoire, on est plus tolérant... » et « on est obligé de respecter l'opinion des autres... ». Enfin, « avec le catch on a réfléchi aux règles de la vie en société... » (Mathieu) et «la négociation est obligatoire pour avancer ... » (Victor).

Les réponses tournant implicitement autour de la violence, nous relançons les élèves au sujet de ce phénomène : « est-ce que le cycle de catch permet de s'interroger sur les phénomènes de violence ? ». Victor pense qu' « on n'est pas toujours le plus fort ou le plus faible... »; "si on est violent on peut aussi être la victime donc on fait d'avantage attention... » (Laura). Quant à Théo, il souligne qu'à travers les techniques de combat mis en scène : «je me suis souvenu de bagarres où j'avais pu donner des coups... Mais la communication peut aussi amener un sentiment de fierté... ». Mathieu déclare qu'il «a bien aimé que les autres ne soient pas des adversaires à 


\section{eJRIEPS 32 avril 2014}

battre ou à éliminer mais que les techniques apprises servent à raconter des histoires de la vie.... ».

Les observations réalisées et les propos tenus au fil des leçons tendent à montrer qu'au travers le catch-scolaire, les jeunes envisagent leurs rôles et se placent dans le groupe de façon différente, mais leurs représentations des relations sociales quittent progressivement le pôle conflictuel pour envisager la possibilité de rapports de coopération, d'écoute mutuelle et d'entraide.

\section{Discussion}

L'action située est un « courant de recherche qui se donne comme objectif l'étude des connaissances et raisonnements pratiques, en situation » (Durand, 1999, p. 3), et qui met en exergue le caractère fortement contextuel de l'activité humaine. Selon le cette perspective les processus cognitifs et l'activité sont indissociables de la situation, c'està-dire que les éléments physiques et sociaux sont signifiants pour l'action du sujet. La mise place du cycle catch s'intègrent dans ce courant et les résultats tendent à les commenter sur deux plans : d'une part sur le versant d'une analyse scientifique et, d'autre part, sur celui d'interprétations de type pédagogique. C'est dans cet ordre que la discussion va maintenant être présentée.

\section{1. L'émotion au cœur du catch-scolaire}

Au cœur du catch-scolaire, l'affectivité apparaît comme composante à part entière de la conduite de l'élève. Au-delà des qualités physiques demandées au cours de la séquence d'apprentissage, de nombreuses influences sont à l'œuvre en relation avec l'histoire et la subjectivité du sujet, avec la manière dont il donne sens au monde extérieur et dont il s'en préserve (Daniel, 2004). Les situations de catch apparaissent ainsi comme un jeu de rôles. En proposant une action conflictuelle vécue corporellement en groupe, le jeu lutté présente de nombreux points communs avec le psychodrame. Dans l'ici et maintenant de la situation motrice suscitée, s'exprime la dynamique voilée du désir inconscient. « Historiquement, le psychodrame est sorti des principes du jeu », avait déjà affirmé Moreno il y a quelques décennies (1965). La prise des rôles, les changements de rôles, l'affrontement des contre-rôles, toute la 


\section{eJRIEPS 32 avril 2014}

dynamique interactionnel dans la mise en jeu corporel trahit une vie fantasmatique intense (Parlebas, 1975). La mise en scène questionne, fait prendre conscience, explore plusieurs solutions face à un problème et les confronte. Le modèle pédagogique utilisé est alors proche du psychodrame où l'adolescent met en œuvre un travail de figuration, d'élaboration psychique souple, et dispose de nombreux moyens de s'exprimer, voire de laisser s'exprimer ce qu'il ne connaît pas de son désir.

Le catch-scolaire nécessite alors de s'intéresser à la manière de créer et d'entretenir des émotions, des sentiments, qui émergent de l'activité et qui sont en mesure de magnifier les conduites d'apprentissage et de faire l'apprentissage du «lien social » (Tisseron, 2010). Comme le notent Ria et Récopé (2005, p.11), les émotions constituent un « ressort pour l'action ». Ainsi, la pertinence des actions motrices et la construction des rôles sociaux passent nécessairement par une maîtrise des réactions affectives et émotionnelles.

Par ailleurs, le respect, la solidarité et la participation fondent une éducation à la citoyenneté (Audigier, 1999) qui permet à chaque élève d'apprendre le respect de la personne, de soi et des autres, le respect du bien commun et du cadre de vie. Pour cela, il est recommandé de mettre en place une démarche active au sein de la classe qui devient ainsi un lieu de pratiques citoyennes. Car la pratique met en action les codes élémentaires de la vie en société, expérimente l'interaction entre plans individuels et collectifs: c'est-à-dire, s'évaluer dans et par le groupe, situer ses pouvoirs et ses limites à l'intérieur d'un cadre reconnu et selon un code commun construit et accepté par tous.

L'investissement de l'élève, l'appropriation des règles et des savoirs sont recherchés à travers une activité signifiante et l'intervention directe des élèves dans la construction des contenus d'apprentissage (Meard \& Bertone, 1996). Le catch-scolaire semble pouvoir contribuer à une meilleure « santé sociale » proche du concept de citoyenneté, mais dépassant le simple respect des règles communes: «On peut définir la citoyenneté de manière plus efficace comme capacité à s'engager positivement dans des projets communautaires : des individus se regroupent, autour d'objectifs communs, 
eJRIEPS 32 avril 2014

ils constituent alors une communauté d'intérêt, soudée par son projet. " (Delignières, 2001, p 11).

La construction, l'exécution, l'évolution des scènes de catch-scolaire demande un élève qui accepte, partage, discute, argumente, critique, coopère, s'investit. Par sa structure singulière, il offre donc une multiplicité d'expériences relationnelles. L'opposition jouée, simulée a provoqué une mise à l'épreuve de l'adaptabilité de l'élève aux différents modèles du lien social ; à ce titre, le catch est susceptible de représenter un outil d'apprentissage de la compétence relationnelle, facteur de diminution du comportement agressif.

L'empathie, que nous avons définie plus haut, passe par l'expérience vécue, par cette dynamique interactionnelle entre pairs, cette capacité à se mettre à la place des autres, comprendre leurs émotions et éviter ainsi l'escalade néfaste de rapports négatifs dominants /dominés (Sironi, 1999). Si le désir de s'opposer, et donc de vaincre ou de dominer, est aussi du ressort culturel des sociétés occidentales, tous les jeux ne sont pas strictement compétitifs dans toutes les sociétés (Lévi-Strauss, 1962). De fait, le plaisir de jouer peut se retrouver dans certains «jeux sportifs déterminés » et des "jeux de rites " si ceux-ci ne sont pas systématisés. Car généralement, le sel de l'ensemble des situations sociales jouées réside dans l'incertitude liée à l'issue du jeu, ou bien dans le déroulement sans cesse changeant et parfois ambivalent (jeux paradoxaux) qui plonge les acteurs dans des émotions bien singulières (Dugas, 2011).

6. 2. Perspectives pédagogiques

Agir, organiser et/ou créer, passer devant les autres, échanger entre spectateurs et acteurs, sont autant de phases qui engagent les élèves dans une activité particulière et qui constituent des séquences que l'on retrouve de façon constante dans chacune des séances tout au long du cycle catch.

La démarche pédagogique utilisée à travers le catch-scolaire réside dans le fait que l'événement vécu ne se réduise pas à une simple pratique motrice pour laquelle la trace resterait implicite, non exploitée, non verbalisée et où l'élève resterait dans le domaine du ressenti, des sensations et impressions. Les échanges verbaux réguliers, type dilemmes moraux entre l'enseignant et le groupe classe sont essentiels (Dionne \& 


\section{eJRIEPS 32 avril 2014}

Saint-Martin, op. cit.). II s'agit d'aider l'élève à se situer par rapport à ce qu'il fait ou dit à propos de la situation ou exercice proposé afin de pouvoir mieux s' exprimer et maîtriser ses réactions émotionnelles et affectives.

Le dilemme moral apparaît central dans le scénario proposé aux élèves et se présente sous forme d'une situation-problème où l'individu est placé face à un choix inévitable qui met en conflit son système de valeurs. Les élèves, témoins ici de la scène d'agression, sont face à un conflit d'obligations: l'objectif est de faire verbaliser les élèves sur la façon dont ils interprètent leur propre conduite. Le catch constituerait alors une médiation pour permettre aux adolescents de s'exprimer sur leurs problèmes d'existence. En somme, il s'agit aussi de sensibiliser à la maîtrise, voire à l'éducation de ses émotions (Goleman, 1995). Autrement dit, on entre alors de plain-pied dans une forme d'éducation aux émotions, plus qu'à leur simple maitrise.

Coopérer, adopter des attitudes d'écoute, d'aide, de tolérance et de respect des autres pour agir ensemble et chercher de nouvelles idées afin de construire et faire évoluer un scénario est indispensable. Aide à l'agresseur, indifférence, aide à la victime (selon plusieurs manières)....l'objectif de formation doit amener à distinguer entre devoir faire et pouvoir ou être capable de faire. L'élève doit distinguer ce qu'il fait de ce qu'il devrait faire.

À partir des jeux de rôles permis par le catch-scolaire, l'élève expérimente les règles de la vie en société, les valeurs qui la fondent et fait quelque peu l'apprentissage de ses droits, de ses obligations et de sa propre responsabilité dans une société démocratique. Les bilans de séquences sont fondamentaux pour développer la tolérance, relativiser les positions personnelles. La verbalisation est ici importante, l'élève reprend, par les mots, les gestes utilisés dans la construction de l'histoire collective.

Le catch offre ainsi aux élèves un espace de liberté où ils peuvent projeter, à travers des situations, leurs angoisses, leurs sentiments. 


\section{eJRIEPS 32 avril 2014}

\section{Conclusion}

L'interaction sujet/situation est indéniablement le point de départ de notre recherche. Celle-ci s'inscrit dans ce rapport consubstantiel entre les élèves agissants et le contexte d'action, en ayant mis à l'épreuve des faits que le catch, absent des séances d'EPS, peut être un support éducatif au sein duquel l'élève, en tant qu'une personne titulaire de droits et d'obligations, s'imprègne du vivre ensemble. Cet apprentissage du jeu social se matérialise en coopérant et en participant, dans l'action, à la réalisation d'objectifs et de projets communs.

Dans cette perspective, la cognition est socialement et culturellement située. " Agir, c'est construire des significations dans un contexte culturel et en relation avec d'autres individus. Ces significations émergent d'un flux d'actions qui ont toujours une dimension sociale, et qui se rattachent à une culture pré-existante " (Saury, Ria, Sève \& Gal-petitfaux, 2006, p. 8). Ainsi, en proposant des situations à l'apparence conflictuelle mais réglementée, pour préserver l'intégrité des sujets, le catch a ici permis - toutes choses égales par ailleurs et avec les précautions d'usage d'une étude ne permettant pas la généralisation du phénomène observé -, de prendre conscience de ses pouvoirs sur les autres, mais aussi de ses propres limites et de vivre des situations de dilemmes moraux en actes (Kohlberg, 1981). Car rappelons qu'un dilemme moral consiste à proposer deux choix qui placent le répondant dans un réel embarras car l'issue choisie ne peut être satisfaisante ou rationalisée.

Sur le plan des compétences scolaires institutionnelles, le catch-scolaire a permis un tant soit peu d'offrir aux adolescents l'occasion de mettre en pratique les compétences 6 et 7 du socle commun où l'objectif " agir et vivre ensemble " affiche clairement la volonté de former de futurs citoyens autonomes et responsables.

Même si les résultats ne s'expriment que dans l'ici et maintenant, ce qui nous conduit à rester prudents sur les ressorts éducationnels d'une telle démarche, il n'empêche que, pour ces élèves, le vécu dynamique du cycle catch a permis une réflexion autour des attitudes et comportements agressifs ou violents.

Cette expérience originale et les résultats obtenus - dans les limites définies précédemment - nous encouragent à poursuivre dans cette voie, en proposant, dans 


\section{eJRIEPS 32 avril 2014}

un futur proche, une recherche qui utilisera la vidéo comme moyen d'observation plus fin des conduites accomplies et qui servira aussi d'outil méthodologique pour des entretiens d'auto confrontation. Cela afin de travailler davantage autour de la notion d'empathie comme aide à la prévention de la violence scolaire, au bien-être relationnel. «Car oser l'autre, c'est développer son empathie, c'est accepter sa différence et, à terme, c'est peut-être réduire la violence sociale. »(Dugas, 2012, p. 184).

\section{Bibliographie}

Albarello, B. (2009). Choisir l'étude de cas comme méthode de recherche. Editions De Boeck dans la collection Méthodes en sciences humaines.

Arborio, A.M., \& Fournier, P. (2010). L'observation directe. Paris : Colin (3 ${ }^{\text {ème }}$ édition). Audigier, F. (1999). L'éducation à la citoyenneté. INRP.

Barbot A. (1989). La construction du combattant de préhension. Qu'est-il important d'apprendre en S.C.P. ? Comment l'observer ? Vers la définition de contenus d'enseignement. In G. Bui-Xuan et coll. (Eds), Méthodologie et didactique de I'E.P.S. (pp. 279-297). Clermont-Ferrand : AFRAPS.

Barthes, R. (1957). Le monde où l'on catche. In Mythologies (p.13-24). Paris : Seuil.

Blanchet, A. \& Gotman, A. (1992). L'entretien. Paris : Nathan.

Bredemeier, B.J., Shields, D.L., Weiss, M.R. \& Cooper B.A.B. (1986). The relationship of sport involvement with children's moral reasoning and aggression tendencies. Journal of Sport Psychologie, 8 (4), 304-318.

Bredemeier, B.J., Shields, D.L., Weiss, M.R. \& Cooper B.A.B. (1987). The relationship between children's legitimacy judgments and their moral reasoning, aggression tendencies, and sport involvement. Sociology of Sport Journal, 4, 48-60.

Brousse, M. (1993). Quel programme en sports d'opposition ? Revue E.P.S., 242, 5052.

Brousse, M. (1994). La technique ou l'ordre du combat. Techniques sportives et éducation physique. Dossier E.P.S., 19, 80-89.

Bui-Xuan, G., (1986). Projection ou tombé ? Biomécanique et société. Revue STAPS, Vol. 7, 13, 13-18. 


\section{eJRIEPS 32 avril 2014}

Bui-Xuan, G (1989). Pédagogie de l'E.P.S et/ou didactique des A.P.S. Enjeux et objectifs. In Bui-Xuan et coll. (Eds), Méthodologie et didactique de l'E.P.S., (pp.41-53). A.F.R.A.P.S.

Butler, J. (2006). Trouble dans le genre. Le féminisme et la subversion de l'identité. Paris : La Découverte.

Caillois, R. (1958). Les jeux et les hommes. Paris : Gallimard.

Callède, J.P. (2007). Rapport annuel du Conseil National des activités physiques et sportives remis au Ministre de la Santé, de la jeunesse et des sports. « $L a$ violence et le sport. Le sport contre la violence. " CNAPS.

Calmet, M. (2006a) Des filles engagées dans les sports de combat mixtes comme le kendo scolaire ? Oui, si le but est moins la victoire que la maîtrise. Les cahiers pédagogiques, 441, 49-50.

Calmet, M. (2006b). Le Kendo-scolaire Une activité physique sportive et artistique Enseigner le kendo-scolaire ou le savoir combattre?, http://www.apcscolaire.fr/IMG/pdf/elements_pour_organiser_Ken_sco.pdf, 2.

Carnel, C., Masschelein., \& Boutoille, O. (2008). Le combat libre : quel(s) effet(s) sur les jeunes ? International Journal of Violence and School, 5, 5-16.

Carrier, C. (1992). Les aspects psychologiques de la pratique du judo chez l'enfant. Actualités Sport et Médecine, 18, 48-51.

Cizeron, M. (2010). Le geste professionnel comme concept et outil d'analyse. In M.

Cizeron et N. Gal-Petitfaux (Eds), Analyse des pratiques : expérience et gestes professionnels (pp. 257-268). Manuscrit auteur.

Coslin, P.G. (2005). La violence à l'adolescence. Les cahiers psychologie politique, 6.

Collard, L. (2004). Sport et agressivité. Méolans-Revel : Déslris.

Daniel, J.F. (2004). Donner du sens aux apprentissages en EPS : le choix de l'APS Judo. In Garsault (Eds), L'EPS en Zone d'Education Prioritaire (pp.81-100). Document Académique Montpellier.

Debarbieux, E. (2008). Les dix commandements de la lutte contre la violence à l'école. Paris : Odile Jacob. 


\section{eJRIEPS 32 avril 2014}

Debarbieux, E. (2011). Le sport, remède ou utopie contre la violence à l'école ? Lemonde.fr, url : http://www.http://www.lemonde.fr/sport/article/2011/10/26.

Debarbieux, E. (2012). Rapport : Le «climat scolaire »: définition, effets et conditions d'amélioration.

Delignières, D. (2001). Sport et santé. Actes du Colloque Sport-Santé Fédération Nationale du Sport en Milieu Rural/Comité Départemental du Sport en Milieu Rural, 10-11.

Dervaux, S. \& Pain, J. (2006). De la violence verbale en milieu scolaire. Spirale, 37, 159-172.

Dionne, J., \& Saint-Martin, N. (2005). Le développement du raisonnement moral. In L. Massé et al. (Eds..), Les troubles du comportement à l'école. Prévention, évaluation, intervention (pp. 281-295). Montréal : G. Morin.

Dugas, E. (2011). Domaines d'action et agressivité motrice en éducation physique scolaire. International Journal on Violence and School, 12, 2-25.

Dugas, E. (2012). L'intégration des publics spécifiques par la mise en jeu corporelle ? In L. Carrier, Collard (dir.), Sport et bien-être relationnel (pp.171-184). Paris : Chiron.

Durand, M. (1999). A propos de l'enseignement en milieu scolaire. Revue EPS 1, 94, 37.

Durand, M. (2001). Chronomètre et survêtement. Reflets de l'expérience quotidienne d'enseignants en éducation physique. Paris : Editions Revue EPS.

Eber, N. (2006). Le dilemme du prisonnier. La découverte : « Repères ».

Eber, N. (2007). Théorie des jeux. Paris : Dunod.

Elias, N., \& Dunning, E. (1994). Sport et civilisation. La violence maîtrisée. Paris: Fayard.

Elias, N. (1976). Le sport, l'état et la violence. Actes de la Recherche en Sciences Sociales, 6, 2-21.

Favre, D. (2007). Transformer la violence scolaire des élèves. Paris : Dunod. 


\section{eJRIEPS 32 avril 2014}

Foster, Y. A. (1997). Brief aïkido training versus karate and golf training and university students scores on self-esteem, anxiety, and expression of anger. Perceptual and Motor Skills, 84, 609-610.

Friedlmeier, W. \& Holodynski, (1999). Emotionale Entwicklung. Heidelberg: Spektrum.

Gal-Petitfaux, N., \& Durand, M. (2001). L'enseignement de l'Education physique comme "action située" : propositions pour une approche d'anthropologie cognitive. Revue STAPS, 55, 79-100.

Goleman, D. (1995). Emotional intelligence. New-York : Bantam book.

Greeno, J. G. (1998). The situativity of knowing, learning and research. American Psychologist, 53(1), 5-26.

Gresser, B. (1992). Lutte, trois mômes et un tapis. Revue EPS, 233, 69-73.

Guerrien, B. (2002). La théorie des jeux. Paris : Economica.

Guilbert, S. (2009). Violences sportives, milieux sociaux et niveaux scolaires Distribution socioculturelle des formes de violence dans le champ des pratiques de terrain. 24-40.

Kirshner, D., \& Whitson, J.A. (1997). Situated cognition: social, semiotic, and psychological perspectives. Mahwah, NJ: Erlbaum.

Kohlberg, L., (1981). Essays on Moral Development, Vol. I : The Philosophy of Moral Development. San Francisco, CA: Harper \& Row.

Lamarre, B.W., \& Nosanchuck, T.A. (1999). Judo, the gentel way : a replication of studies on martial arts and aggression. Perceptual and motor Skills, 88, 99299.

Lave, J. (1988). Cognition in practice. Cambridge: Cambridge University Press.

Lévi-Strauss, Cl. (1962). La Pensée sauvage. Paris : Plon.

Loizon, E. (2008). Analyse des pratiques d'enseignement du judo en EPS. Revue eJERIEPS, 14, 63-82.

Louveau, C., \& Davisse, A. (1998). Sport, école, société : la différence de sexe. Paris : L'Harmattan.

Loyer, F. (2010). Histoire de la lutte et du catch en France. Caen : PUC. 
eJRIEPS 32 avril 2014

Loyer, F. (2013). Le catch et la lutte entre certitude et incertitude : quelle pratique de combat pour le Ille millénaire ? Revue Staps, 99, 49-59.

Marzano, M. (Dir., 2011). Dictionnaire de la violence. Mercuès : Qualibris France Quercy (Quadrige, PUF).

Mauss, M. (1966). Les techniques du corps. Sociologie et anthropologie. Paris : PUF.

Meard, J., \& Bertone, S. (1996). L'élève qui ne veut pas apprendre en EPS. Revue EPS, 259, 61-64.

Meirieu, Ph. (2008). Adolescent à l'école: est-ce possible? Cultures adolescentes. Paris : Autrement.

Meirieu, Ph. (2009). Quelle parole contre la violence ? Les Mercredi de Créteil, Créteil, 21 janvier 2009.

Moatti, D. (2000). La communication par la violence. Communication et langages, 123, 80-96.

Morhain, Y. (2001). Malaise social et violences d'adolescents. Cahiers de psychologie clinique, 16, 79-86.

Moreno, J. (1965). Psychothérapie de groupe et psychodrame. Paris : PUF.

Miermont, J. (2008). Violence et adolescence : réflexions écosystémiques. Le Journal des psychologies, 263, 32-35.

Mutz, M., \& Baur, J. (2009). The role of sports for violence prevention : sport club participation and violent behaviour among adolescents. International Journal of Sport Policy, 1, 3, 305-321.

Nosanchuck, T.A. (1981). The way of the warrior: the effects of traditionnal arts trainings on aggressiveness. Agressive Behavior, 15, 153-159.

Pain, J., \& Hellbrunn R. (1986). Intégrer la violence. Vigneux : Matrice.

Pain, J. (1992). Écoles : violence ou pédagogie. Vigneux : Matrice.

Parlebas, P. (1975). Piste noire : Jeu sportif, rêve et fantaisie. Revue esprit, l'éducation physique.

Parlebas, P. (1986). Eléments de sociologie du sport. Paris : PUF.

Pfister, R. (1986). Le sport et la catharsis de l'agressivité. La psychopédagogie des activités physiques et sportives. Toulouse : Privat. 
eJRIEPS 32 avril 2014

Rimé, B. (2005). Le Partage social des émotions. Paris : PUF.

Reynes, E., \& Lorant, J. (2003). Judo, agressivité et maîtrise de la colère : étude longitudinale chez des enfants de 8 ans. Staps, 60, 93-105.

Ria, L., \& Récopé, B. (2005). Les émotions comme ressort de l'action. In L. Ria (Ed.), Les émotions (pp. 11-30). Paris : Éditions EPS

Santschi, A. (1993). Le judo et les enfants. Paris : Macolin.

Saury, J., Ria, L., Sève, C., Gal-petitfaux, N. (2006). Action ou cognition située. Revue EPS, 321, 5-11.

Schubauer-Leoni, M.-L. (2008). La construction de la référence dans l'action conjointe professeur-élève. In N. Wallian, M.-P. Poggi \& M. Musard (Eds.), Co-construire des savoirs: les métiers de l'intervention par les APSA (pp. 67-85). Besançon : PUFC.

Sensevy, G. (2007). Des catégories pour décrire et comprendre l'action didactique. In G. Sensevy \& A. Mercier (Eds.), Agir ensemble : l'action didactique conjointe du professeur et des élèves (pp. 174-177). Rennes : PUR.

Simar, C. Jourdan, D. (2008). La contribution spécifique de l'éducation physique à la lumière de travaux de recherche récents. Revue EPS, 329, 10-12.

Sironi, F. (1999). Bourreaux et Victimes. Psychologie de la torture. Paris : Odile Jacob.

Smith, P.K. (2002). Violence in Schools: the response in Europe. London : Routledge Falmer.

Terrisse, A. (1996). Analyse de la transposition didactique en judo : évolution du "savoir combattre" dans l'enseignement du judo à l'école à travers la revue EPS de 1950 à 1993. Revue française de pédagogie, 116, 1, 65-75.

Terrisse, A. \& Carnus, M-F. (coordonné par). (2009). Didactique clinique de l'éducation physique et sportive. Quels enjeux de savoirs ? Bruxelles : De Boëck.

Tisseron, S. (2010). L'Empathie, au cœur du jeu social. Paris : Albin Michel.

Wargo, M., Spirrison, C., Thorne, M., \& Henley, T. (2007). Personality characteristics of martial artists. Social Behavior and Personality, 35, 3, 399-408. 